Meta

Journal des tradlucteurs

Translators' Journal

\title{
La voix passive en français et sa traduction en espagnol
}

\section{Valentín García Yebra}

Volume 35, numéro 3, septembre 1990

La traduction dans le monde hispanolusophone

URI : https://id.erudit.org/iderudit/004630ar

DOI : https://doi.org/10.7202/004630ar

Aller au sommaire du numéro

\section{Éditeur(s)}

Les Presses de l'Université de Montréal

\section{ISSN}

0026-0452 (imprimé)

1492-1421 (numérique)

Découvrir la revue

Citer cet article

García Yebra, V. (1990). La voix passive en français et sa traduction en espagnol. Meta, 35(3), 510-517. https://doi.org/10.7202/004630ar d'utilisation que vous pouvez consulter en ligne.

https://apropos.erudit.org/fr/usagers/politique-dutilisation/ 


\section{LA VOIX PASSIVE EN FRANCAIS ET SA TRADUCTION EN ESPAGNOL}

VALENTIN García YeBra

Espagne

1. «Passive» se dit de la fonction du sujet qui ne réalise pas mais qui subit ou reçoit l'action signifiée par le verbe dont il est sujet grammatical. On appelle également passive la forme verbale et la proposition où cette fonction est exprimée.

2. Dans les langues romanes, la forme passive spécifique est celle appelée voix passive, forme verbale composée du participe passé du verbe qui exprime l'action et, comme auxiliaire, du temps correspondant du verbe être (ou son équivalent dans chaque langue). C'est à proprement parler une forme verbale périphrastique et on ne lui applique pas rigoureusement le nom de voix, qui correspond exactement à la forme passive latine quand il s'agit d'un seul mot aux désinences particulières: amaris (tu es aimé[e]), amabuntur (ils seront aimés, elles seront aimées).

3. Il est également possible d'exprimer la fonction passive en faisant précéder le verbe transitif du pronom se: ce plat se mange froid, este plato se come frío; il y a des choses qui ne se font pas, hay cosas que no se hacen.

4. Nous venons d'exposer des notions de grammaire élémentaire sur lesquelles il est inutile d'insister. Du point de vue du traducteur, le plus intéressant est le fait de connaître les différences d'usage des voix verbales passives dans les deux langues impliquées dans la traduction. N'importe quel traducteur se servant du français comme langue d'origine et de l'espagnol comme langue terminale doit savoir que, bien qu'employée moins fréquemment qu'en anglais, la voix passive est utilisée en français plus souvent qu'en espagnol.

5. En effet, l'espagnol tend à éviter la voix passive en l'employant presque exclusivement quand des raisons spéciales, de clarté ou de rythme par exemple, déconseillent l'usage de la voix active. Par conséquent, lorsqu'on traduit du français en espagnol, il convient en principe de remplacer la voix passive par la voix active. Pour cela, le traducteur doit bien connaitre le fonctionnement de la voix passive en espagnol ainsi que les possibilités de cette substitution 1 .

6. À la fin du XVIIIe siècle, Joseph de Covarrubias, dans la préface de sa traduction des Aventures de Télémaque, de Fénelon (Madrid, 1797, p. 96), signalait la désaffection de l'espagnol pour la voix passive, comparativement à l'usage qu'en faisait la langue latine: La otra razón de diferencia se demuestra en la preferencia que damos a las oraciones activas sobre las pasivas. Las pasivas eran numerosas entre los Latinos; decian: a me Caesar quotidie visebatur. «Yo visitaba a César todos los días ${ }^{2} . »$

En latin, on utilisait assez fréquemment la voix passive, même en poésie, comme dans ces deux vers d'Ovide:

Nec tantum segetes alimentaque debita dives

Poscebatur humus (Metam. I, 136-137.)

Construction impossible en espagnol, qui pourrait, au maximum, avoir ici recours à la forme pronominale à sens passif : 
Y no sólo mieses y alimentos debidos se pedían A la tierra fecunda ${ }^{3}$.

7. Les traducteurs ne doivent pas se laisser influencer par le mauvais usage de la voix passive que l'on fait parfois dans la presse. Certains textes montrent une influence plus ou moins directe de l'anglais. En voici deux exemples :

(...) balcones, aleros, salientes de muros y tuberías que pueden ser fácilmente trepados por los delincuentes. $(A B C, 04 / 07 / 85$, p. 57, col. 3.)

La niña transplantada de pulmones y corazón cumple mañana catorce años. (Ibid., $05 / 12 / 86$, p. 45 , col. 1 et 2 .)

Dans le premier, on met à la voix passive un verbe intransitif, ce qui est inadmissible en espagnol (la construction correcte serait: «(...) por los cuales pueden trepar fácilmente los delincuentes»). Dans le deuxième, on écarte le verbe de son objet direct: pulmones y corazón, remplacé par l'objet indirect: la niña (dans une construction active : han transplantado a la niña pulmones y corazón; à la voix passive : pulmones y corazón han sido transplantados a la niña; dans une forme pronominale à sens passif: se han transplantado a la niña pulmones y corazón).

8. La répugnace de l'espagnol à l'égard de la voix passive ressort d'une façon inconsciente dans les lignes suivantes, écrites par un journaliste connu:

Hasta ahora, los magistrados de este altísimo Tribunal [Tribunal Constitucional] eran tratados con todos los remilgos, como a vestales que custodian el sagrado de la Constitución ${ }^{4} . »$ (J. Campmany, $A B C, 18 / 10 / 83$, p. 17, col. 3.)

La préposition $a$ devant vestales, aussi bien si elle provient réellement de Campmany que si elle a été ajoutée par le photocompositeur, montre la prédilection de l'espagnol à l'égard de la voix active: le début du texte fait ressortir los magistrados, qui occupent la première place après l'expression adverbiale de temps et avant le verbe, à la voix passive.

Or, l'auteur ou le photocompositeur, attiré sans s'en rendre compte par la construction active, établit la comparaison comme si l'on avait écrit:

(...) les magistrats (...) on les choyait, comme on choierait des vestales qui (...).

(...) a los magistrados (...) los trataban con todos los remilgos, como (se trataria) a vestales que (...).

9. Par ailleurs, on ne saurait justifier en espagnol l'usage de la voix passive tout simplement par le désir de rehausser des concepts qui exerceraient normalement dans la construction active la fonction de complément direct et qui, par conséquent, auraient tendance à occuper un lieu voilé à l'intérieur du texte. C'est pour cela que cette phrase d'un critique actuel, bien que correcte, est ingrate:

Ciudades, campos, edificios, monumentos y hombres son hechos emerger casi desde la nada por el creador. (M. Ga Posada, ABC Literario, 06/12/86, p. III, col. 1.)

Taduction littérale: Villes, champs, bâtiments, monuments et hommes sont faits émerger presque du néant par leur créateur.

Par contre, une construction active semblable à celle qui suit ne choquerait pas :

Ciudades, campos, edificios, monumentos y hombres emergen casi de la nada por obra de su creador. 
Ou bien cette autre, un peu plus dure, mais aussi acceptable:

Ciudades, (...) y hombres, los hace emerger su creador casi de la nada.

10. En somme, comme on l'a déjà dit dans le paragraphe 5, l'espagnol utilise la voix passive presque exclusivement quand des raisons spéciales, telles que la clarté ou le rythme, déconseillent l'usage de la voix active. Nous en verrons par la suite certains exemples.

11. Les traductions des textes suivants (citées par M. Wandruszka, Sprachen vergleichbar und unvergleichlich, trad. Nuestros idiomas, comparables e incomparables (Madrid, 1976, p. 625) ne sont donc pas de bonnes traductions:

1. Nommé ministre de l'Éducation nationale, Abel Bonnard blâma la tiédeur de ses prédécesseurs, il réclama que l'Université s'engageât; il ne fut pas suivi (...) (Simone de Beauvoir, La Force de l'âge, Paris, 1960, p. 528.)

Nombrado ministro de Educación Nacional, Abel Bonnard criticó la tibieza de sus predecesores, reclamó que la Universidad «se comprometiera»; no fue seguido (...) (Trad. de Silvina Bullrich, Buenos Aires, 1961.)

2. L'ordre de rendre les armes a été donné avant-hier. (André Malraux, La Condition humaine, Paris, 1947, p. 282.)

La orden de entregar las armas fue dada anteayer. (Trad. de César A. Comet, Buenos Aires, 1958.)

Dans 1, si l'on ne tient pas compte de la juxtaposition des deux propositions principales: «(...) criticó la tibieza de sus predecesores, reclamó que la U. se comprometiera» (l'espagnol demande ici la coordination : «criticó (...) y reclamó que (...)») ainsi que de la ponctuation (le point-virgule après comprometiera devrait être un point ou un deux points), il y a un calque évident de la construction passive: no fue seguido. Il serait plus naturel et, par conséquent, mieux, d'écrire: No tuvo seguidores, No halló quien lo siguiera ou quelque chose de semblable. Dans 2 on aurait pu éviter la forme passive périphrastique, qui est ici aussi un calque syntaxique de l'original, en ayant recours à la forme pronominale à sens passif : «La orden (...) se dio anteayer.»

12. Je commenterai par la suite le résumé d'un article publié par Jean Dubois dans le Journal de psychologie, janvier-mars 1966, tel qu'il apparaît dans le Grand Larousse de la langue française, Paris, 1976, p. 4064 a.

Quatre facteurs de la construction passive en français y sont mis en relief. Je vais les énoncer, et je proposerai pour chaque exemple français la traduction espagnole qui me semble la plus naturelle et qui, par conséquent, pourrait en être, à mon avis, le meilleur équivalent ${ }^{5}$.

1) Dans la phrase active, la logique naturelle demande comme sujet un être animé et, comme objet, un être inanimé (Jacques a cassé la cruche : Jacques ha roto el cántaro). Par conséquent, on donnera la préférence à la construction passive quand on voudra présenter un être animé comme but du processus verbal attribué à un objet inanimé. On ne dira donc pas: Une voiture a renversé un passant (voix active), Un coche ha atropellado/atropelló6 a un peatón, mais :

Un passant a été renversé par une voiture.

Un peatrón ha sidolfue atropellado por un coche.

En espagnol, la logique naturelle est probablement moins décisive qu'en français, et d'autres raisons pourraient influencer ce choix, par exemple le désir d'éviter la cacophonie des hiatus coche ha atropellado a. Mais si nous remplaçons la phrase par cette autre, dans laquelle l'agent est una roca desprendida de la montaña (un rocher détaché de 
la montagne), le verbe, matar (tuer), et celui qui subit l'action, un excursionista (un excursionniste), il est presque sûr que la construction espagnole se ferait à la voix active:

Una roca desprendida de la montaña mató a un excursionista

et non pas

Un excursionista fue muertolmatado por una roca desprendida de la montaña

ce à quoi contribuerait bien l'hésitation entre les participes matado et muerto. Même pas au cas où l'excursionniste serait notre ami, nous ne dirions: Nuestro amigo fue muerto/matado por ..., mais Una roca ... mató a nuestro amigo. Ou bien, si nous voulons souligner la priorité de la personne : A nuestro amigo lo mató una roca ${ }^{7}$...

2) La fonction du sujet est normalement exercée par l'être animé ou l'objet connu au sujet duquel on va émettre un jugement ou une information. Par conséquent, on préférera la construction passive si l'on veut qu'un être animé ou une chose, déterminés par le contexte, apparaissent comme récepteurs de l'action exprimée par le verbe:

$\mathrm{X}$ est un préfet actif. Ses mérites ont été hautement appréciés par le gouvernement. (Le Monde, 30 avril 1965.)

La traduction: «X es un prefecto activo. Sus méritos han sido muy apreciados por el gobierno» sera sans doute inférieure à cette autre: «X es un prefecto activo. El gobierno ha apreciado mucho sus méritos.» Or, cette règle ne contredit-elle pas à un certain point la précédente? Les "mérites» ne sont pas des êtres animés, mais des valeurs abstraites, tandis que le gouvernement, bien que ce soit également une entité abstraite, est constitué par des êtres humains, de sorte qu'il pourrait être remplacé par «les gouvernants.» 3) Quand l'un des deux termes de la relation établie par le verbe est au singulier et l'autre au pluriel, la norme veut que le sujet soit au singulier. On économise ainsi une marque du pluriel dans le verbe, et on évite fréquemment une ambiguïté quant à la portée de la relation; par conséquent, la voix passive est préférée si le terme qui subit ou reçoit l'action est au singulier. Exemple:

Un propriétaire terrien a été enlevé vendredi par six hommes. (Le Monde, 30 avril 1965.)

Un terrateniente fue secuestrado el viernes por seis hombres.

La construction active: «Six hommes ont enlevé un propriétaire terrien» pourrait, nous dit-on, être ambiguë (chacun un? ce qui nous donnerait six propriétaires terriens enlevés). Cependant, en espagnol on pourrait préférer l'énoncé suivant: «Seis hombres secuestraron el viernes a un terrateniente», et personne ne serait tenté de penser que chacun de ces six hommes avait enlevé un propriétaire terrien.

4) L'économie du discours exige fréquemment l'inclusion d'une phrase dans l'autre en utilisant un pronom relatif. Si celui qui est représenté par ce pronom est l'être animé ou la chose qui subit l'action signifiée par le verbe de la proposition relative, la phrase incluse adopte souvent la forme passive pour conserver l'ordre normal sujet-verbe:

Les hornmes politiques qui ont été consultés hier par le chef de l'État se sont montrés très réservés.

C'est pour cela, conclut cette norme, que la voix passive est plus fréquente dans les propositions relatives que dans les principales.

On pourrait également éviter ici la voix passive en espagnol, en traduisant:

Los hombres políticos a quienes consultó ayer el jefe del Estado se mostraron muy reservados. 
Cependant, il convient de remarquer que la voix passive existe aussi en espagnol, et qu'elle ne doit pas être évitée à tout prix, mais uniquement lorsqu'elle ne présente aucun avantage sur la voix active. Dans les propositions relatives auxquelles cette règle de la construction française se rapporte, une bonne solution pourrait consister à conserver la voix passive tout en omettant le relatif et l'auxiliaire:

Los hombres políticos consultados ayer por el jefe del Estado (...)

La brièveté sans perte de sens est également recommandable dans la traduction.

13. L'auteur de l'article du Grand Larousse de la langue française ajoute à ceux qui ont été énumérés par Jean Dubois les deux facteurs suivants :

5) le volume des termes, en raison duquel on place derrière le verbe les membres les plus longs, les termes coordonnés :

La Grande-Bretagne est composée de l'Angleterre, du pays de Galles, de l'Écosse et de l'Irlande du Nord.

Voilà un exemple de construction passive discutable. Si est composée était une forme verbale vraiment passive, ce serait un présent; l'action signifiée par le verbe serait encore en cours de réalisation, comme il arriverait dans cette autre phrase :

La Grande-Bretagne est visitée par beaucoup d'étrangers.

Mais est composée, dans le texte en question, signifie un état, non pas une action qui est en train d'être réalisée. La construction est semblable à celle utilisée par César au début de la Guerre des Gaules: Gallia est omnis divisa in partes tres, où, évidemment, est divisa n'est pas un présent passif, qui a en latin, une forme propre et synthétique : dividitur; ce n'est pas non plus un prétérit parfait, que nous traduirions par fut divisée (fue dividida). Ni dans est divisa, ni dans est composée, la forme verbale est ne fonctionne comme auxiliaire de la voix passive, mais comme verbe attributif; le caractère passif réside exclusivement dans les participes passés employés adjectivement, composée et divisa. Il en arriverait de même dans Madrid está situada aproximadamente en el centro de España, où ser est remplacé par estar comme verbe attributif. Aussi, dans la traduction espagnole de l'exemple du GLLF, on n'utiliserait pas comme auxiliaire ser, mais estar:

La Gran Bretaña está compuesta por Inglaterra, el País de Gales, Escocia e Irlanda del Norte.

tandis que dans l'exemple où nous trouvons est visitée nous traduirions : La G. B. es visitada... A part cela, está compuesta pourrait être remplacé, sans aucun désavantage, par se compone, et la dernière phrase pourrait mieux être construite à la voix active:

Visitan la Gran Bretaña muchos extranjeros.

6) Quand le contexte demande un imparfait du subjonctif, la passivisation permet fréquemment de remplacer des formes plus périmées par fût ou fussent:

Il serait utile que sa demande fût présentée aussi par nous. (Discours présenté par Marcel Cohen; l'orateur évite «que nous présentassions».)

Il s'agit là, en effet, d'une raison valable pour construire une phrase française à la voix passive. Nous avons déjà dit qu'en espagnol on peut également avoir recours à la voix passive pour éviter une cacophonie. Mais, en espagnol, l'imparfait du subjonctif 
n'est généralement pas plus cacophonique que la propre voix passive. L'exemple apporté par M. Cohen pourrait en effet être très bien traduit en mettant le verbe à la voix active :

Sería conveniente que también nosotros presentásemos su petición.

14. L'un des grands avantages que l'on attribue d'habitude à la voix passive est celui d'exprimer un événement sans en mentionner l'agent, qui peut bien être inconnu. Le GLLF apporte les deux exemples suivants auxquels nous ajoutons une traduction mot à mot:

Un bijoutier a été assassiné rue Gambetta.

Un joyero ha sido asesinado en la calle Gambetta.

(On ne connaît pas l'auteur de l'assassinat);

Le pain a encore été augmenté.

El pan ha sido nuevamente aumentado (de precio).

On y observe que les deux phrases peuvent devenir actives si l'on utilise le pronom indéfini on pour représenter l'agent :

On a assassiné un bijoutier.

On a encore augmenté le pain.

En espagnol, les énoncés les plus naturels des contenus des deux phrases et, par conséquent, leur meilleure traduction, seraient:

Han asesinado a un joyero.

El pan ha vuelto a subir /... ha subido de nuevo.

Dans le premier, on a recours à l'usage de la troisième personne du pluriel sans sujet pour exprimer une action impersonnelle; il faut remarquer que le verbe est au pluriel même si l'on considère que l'agent a été probablement unique, comme lorsqu'on dit «han llamado a la puerta" (on a sonné/frappé à la porte); nous savons que l'action de sonner ou de frapper avec le heurtoir a été réalisée par une seule personne, même si elle peut être accompagnée par une autre ou par d'autres. Dans la deuxième phrase, nous remplaçons l'usage transitif d'augmenter par l'usage intransitif de subir, et l'objet ou complément direct de la construction active française devient sujet.

15. Dans un alinéa titré PASSIFS COMPLEXES, le GLLF inclut plusieurs exemples dans lesquels la construction active serait en espagnol plus naturelle que la construction passive, ou qui rejetteraient totalement la construction passive, par exemple la structuration d'un groupe verbal composé de auxiliaire + infinitif, qui peut devenir passif par la passivisation de l'infinitif:

On peut (doit, va, vient de) couper cette route.

Cette route peut (doit, va, vient d') être coupée.

La construction la plus naturelle en espagnol serait:

Pueden (deben, van a, acaban de) cortar esta carretera

par l'usage impersonnel du verbe en troisième personne du pluriel quand on ne connaît pas le sujet ou qu'on ne veut pas le nommer. 
La construction passive de la phrase

Paul a été prié de sortir

est justifiée en français, mais serait inadmissible en espagnol:

Paul ha sido rogado de salir.

Paul ha sido rogado que salga.

Par contre, les constructions suivantes seraient correctes:

Han rogado a Paul que salga.

A Paul le han rogado que salga.

Ou même :

Se ha rogado a Paul que salga. simple:

Et, en supposant que le passé composé doive être traduit par le pretérito perfecto

Rogaron a Paul que saliera.

A Paul le rogaron que saliera.

Se rogó a Paul que saliera.

Il serait impossible en espagnol de construire à la voix passive les trois phrases suivantes, parfaitement admissibles en français :

Le château n'était pas achevé de meubler. (Chateaubriand)

Ma deux-chevaux n'est pas finie de roder.

Mạ robe est commencée de couper.

El castillo no era acabado de amueblar.

Mi dos caballos no es terminado de rodar.

Mi vestido es comenzado a cortar.

Le caractère passif de était achevé et est finie dans les première et deuxième phrases est aussi discutable que celui de est composée dans l'exemple relatif à la composition de la Grande-Bretagne, dans l'alinéa 13. Une preuve contraire est constituée par le fait qu'ici également la phrase espagnole remplacerait avantageusement l'auxiliaire ser par estar: El castillo no estaba acabado de amueblar, Mi dos caballos no está terminado de rodar, qui ne seraient pas non plus des solutions satisfaisantes. Les trois phrases pourraient être bien traduites en les construisant à la voix active :

No se había acabado de amueblar el castillo.

No he han terminado de rodar mi dos caballos.

Helhan comenzado a cortar mi vestido.

Finalememt, en français, la forme passive dans la construction unipersonnelle est admise par certains verbes transitifs indirects, comme parler de, procéder à, reprocher de:

Il a été parlé de toi à la dernière réunion.

Il sera procédé au plus tôt à l'inhumation.

Il est reproché à cet homme d'avoir volé un pain.

GLLF, 4063a 
La construction passive équivalente serait impossible en espagnol. Il faudrait procéder à la transformation active, qui peut se faire en francais avec on: On a parlé..., On procédera..., On reproche..., et, en espagnol, avec se comme sujet indéterminé:

Se habló de ti en la última reunión.

Se procederá a la inhumación lo más pronto posible.

Se reprocha a este hombre haber hurtado un pan.

\section{CONCLUSION}

Le traducteur doit conserver intégralement le contenu de l'original, sans rien ajouter ni rien ôter. Mais cela ne suffira pas pour obtenir la meilleure traduction possible. Pour atteindre ce but, il ne suffit pas de considérer «ce que» 1'on traduit, mais aussi «comment» on le traduit.

Le contenu d'une proposition passive peut être reproduit en toute exactitude au moyen d'une proposition active. La signification de la phrase latine du texte de Covarrubias cité dans l'alinéa 6, «a me Caesar quotidie visebatur», est tout aussi conservée dans «César era visitado por mí todos los días» que dans «Yo visitaba a César todos los días». Mais conserve-t-on également dans l'une et l'autre version le style de la phrase latine?

Il faut distinguer dans l'original le style de l'auteur et le style de la langue. On doit reproduire, dans la mesure du possible, le style de l'auteur. Par contre, on doit se passer du style de la langue d'origine et adapter la traduction au style de la langue terminale. Or, l'un des traits caractéristiques du style de l'espagnol par rapport à celui d'autres langues, parmi elles le français, est le fait de préférer la voix active. Mais attention: préférer une chose ne veut pas dire exclure l'autre. En espagnol, il existe aussi la voix passive. Est-il possible d'établir des normes pour l'usage d'une voix ou de l'autre? La seule norme possible a un caractère négatif : la voix passive française doit être traduite par la voix active espagnole quand il n'y a pas de raisons spéciales conseillant le contraire: la clarté, le rythme, le souci d'éviter une cacophonie... peuvent constituer des raisons en faveur de la voix passive. La capacité de percevoir ces raisons et de leur accorder chaque fois leur juste valeur fait partie du talent du traducteur.

\section{NOTES}

1. On peut trouver un exposé sur ce sujet, fait d'une façon claire et concise, dans le Diccionario de uso del español, de María Moliner, s. vv. pasivo (p. 65 a et b) et verbo, b) Forma pasiva perifrástica (p. 1500 a et b). Ce dictionnaire constitue par ailleurs un instrument de travail indispensable à ceux qui traduisent de n'importe quelle langue en espagnol.

2. L'autre raison de différence est démontrée par la préférence que nous accordons aux propositions actives par rapport aux propositions passives. Les propositions passives étaient nombreuses chez les latins; ils disaient : A me Caesar quotidie visebatur (Je rendais visite à César tous les jours).

3. «Et non seulement la moisson et les aliments dus étaient réclamés à la terre féconde.»

4. «Jusqu'à présent, les magistrats de cette très haute Cour (Cour constitutionnelle) étaient choyés comme des vestales qui gardent le temple de la Constitution.»

5. Je reproduis ici, avec de légères modifications, le contenu des pages 231-237 de mon livre Teoría y práctica de la traducción, 2e éd. révisée, Gredos, Madrid, 1984.

6. Sur la traduction espagnole du «passé composé» français, cf. mon livre déjà cité : Passé simple y passé composé. Su traducción al español, pp. 169-181.

7. La possibilité de recueillir anaphoriquement, au moyen d'un pronom personnel, le complément direct placé devant, rend facile l'usage de la voix active sans perdre le relief que l'on accorderait, dans la construction passive, au même concept transformé en sujet. 